\title{
Levantamento florístico de um remanescente de Mata Atlântica no litoral norte do Estado da Bahia, Brasil
}

\author{
Maria Alves $^{1,2,5}$, Rodrigo Bruno Oliveira ${ }^{3}$, Suzane Rocha Teixeira ${ }^{4}$, Maria Lenise Silva Guedes ${ }^{4}$ e \\ Nádia Roque ${ }^{4}$
}

Recebido: 7.01.2015; aceito: 1.06.2015

\begin{abstract}
Floristic survey from an Atlantic Forest remnant on the northern coast of Bahia State, Brazil). The Atlantic Forest in the Northeastern Brazil is composed by several physiognomies, with about $46 \%$ of its remnants located in the State of Bahia. However, floristic inventories conducted in the Atlantic Forest in the State are mostly focused in areas on the southern coast and vegetation dunes on the northern coast. Thus, this study aimed at a floristic survey of a semideciduous forest remnant located at the Fazenda Regional de Criação/UFBA, Entre Rios municipality, on the northern coast of the State. We recorded 192 species belonging to 139 genera and 59 were families. The presence of two new species, one rare species, four species restricted to this region, and two cited as vulnerable reinforce the importance of inventories in the Atlantic Forest remnants in the northern coast of Bahia, since they present high species richness and have physiognomic features not found in other areas of the Atlantic domain.
\end{abstract}

Keywords: Atlantic Forest, Checklist of Angiosperms, Entre Rios, Semideciduous Forest

RESUMO - (Levantamento florístico de um remanescente de Mata Atlântica no litoral norte do Estado da Bahia, Brasil). A Mata Atlântica do Nordeste brasileiro abriga diversas fisionomias, tendo cerca de $46 \%$ dos seus remanescentes localizados no Estado da Bahia. Entretanto, inventários florísticos realizados no Domínio Atlântico no Estado estão geralmente concentrados em áreas no litoral sul e de restinga no litoral norte. Dessa forma, o presente trabalho realizou o levantamento florístico de um remanescente de Floresta Estacional Semidecidual, na Fazenda Regional de Criação/UFBA, município de Entre Rios, litoral norte do Estado. Foram registradas 192 espécies, distribuídas em 139 gêneros e 59 famílias. A presença de duas espécies novas, uma espécie rara, quatro espécies com distribuição restrita para essa região e duas citadas como vulneráveis demonstram a importância dos remanescentes de Mata Atlântica do litoral norte da Bahia, visto que eles possuem riqueza e características fisionômicas não encontradas em outras áreas do Domínio Atlântico.

Palavras-chave: Checklist de Angiospermas, Entre Rios, Floresta Atlântica, Floresta Estacional Semidecidual

\section{Introdução}

A Mata Atlântica do Nordeste brasileiro abriga formações pioneiras, porções de Floresta Ombrófila, Floresta Estacional Semidecidual e Decidual, ocupando hoje cerca de $2,21 \%$ de seu território original (Tabarelli et al. 2006). Pouco se conhece sobre as florestas estacionais no Nordeste (Rodal et al. 2005), apesar de elas serem o segundo componente em importância no Domínio Atlântico (DA), antecedidas somente pelas Florestas Ombrófilas (MMA 2014).

Segundo Tabarelli et al. (2006), mais de 46\% dos remanescentes de Mata Atlântica mapeados no Nordeste estão localizados na Bahia. Contudo, grande parte dos inventários florísticos estão concentrados na porção sul do Estado, em florestas ombrófilas ou áreas transicionais (Amorim et al. 2005, Amorim et al. 2008, Amorim et al. 2009, Thomas et al. 2009,

1. Trabalho de Conclusão de Curso da primeira Autora

2. Universidade Estadual de Feira de Santana, Departamento de Ciências Biológicas, Programa de Pós-Graduação em Botânica, Km 3, BR 116, 44031-460 Feira de Santana, Bahia, Brasil

3. Universidade Estadual de Santa Cruz, Programa de Pós-Graduação em Ecologia e Conservação da Biodiversidade, Campus Soane Nazaré de Andrade, Rodovia Jorge Amado, Km 16, Bairro Salobrinho, 45662-900 Ilhéus, Bahia, Brasil

4. Universidade Federal da Bahia, Instituto de Biologia, Departamento de Botânica, Rua Barão de Geremoabo s/n, Campus Universitário de Ondina, 40171-970 Salvador, Bahia, Brasil

5. Autor para correspondência: maria.alves1987@hotmail.com 
Coelho \& Amorim 2014), com apenas um estudo na porção central (Carvalho-Sobrinho \& Queiroz 2005) e quatro outros no litoral norte, em fisionomia de restinga (IBGE 2004, Queiroz et al. 2012, Silva \& Menezes 2012, Gomes \& Guedes 2014).

O litoral norte baiano é considerado uma das áreas focais do Corredor de Mata Atlântica do Nordeste, que está em fase de implementação (Amane 2014). Segundo Gomes \& Guedes (2014), é possível perceber que a biodiversidade da região é grande, com potencial para ser ainda maior, devido ao pequeno esforço amostral. Sendo assim, com o intuito de aumentar o conhecimento sobre florestas estacionais e a flora do litoral norte do Estado, o presente trabalho teve como objetivo realizar o levantamento florístico de um remanescente de Floresta Estacional Semidecidual localizado no município de Entre Rios, Bahia.

\section{Material e métodos}

O estudo foi realizado na Fazenda Regional de Criação (FRC), Universidade Federal da Bahia (UFBA), município de Entre Rios, litoral norte da Bahia, entre as coordenadas $11^{\circ} 55^{\prime} 20^{\prime \prime}$ S e $38^{\circ} 08^{\prime} 42^{\prime \prime} \mathrm{W}$. A área possui cerca de 200 ha de remanescente de Mata Atlântica, que correspondem a aproximadamente $56 \%$ de sua área total (figura 1). O remanescente é um trecho de mata contínua, com clareiras provocadas pelo corte seletivo de madeira. Aárea é circunvizinha à comunidade do Distrito de Aguazinha, onde existem casas, plantações e pasto para criação de gado, além da plantação de eucalipto e estradas automotivas de terra.

Para o levantamento foram realizadas 14 expedições de coleta entre agosto de 2009 e fevereiro de 2011. Todos os indivíduos férteis (flor e/ou fruto) foram coletados através de caminhamento pelas trilhas pré-existentes. As identificações foram feitas com base em literatura especializada e consulta a especialistas. O sistema de classificação utilizado foi o proposto pela APG III (APG 2009), e a descrição dos hábitos seguiu Radford et al. (1974). Os dados de distribuição de espécies foram retirados da Lista de Espécies da Flora do Brasil (2015). Os espécimes coletados foram herborizados e inseridos no herbário Alexandre Leal Costa (ALCB) do Instituto de Biologia da Universidade Federal da Bahia, com duplicatas enviadas ao Smithsonian Institution (US) e ao herbário do Museu Botânico de Curitiba (MBM) (acrônimos conforme Thiers 2015).

Para a confecção do mapa de localização da área de estudo foi utilizado ArcGIS software (ESRI 2010).

\section{Resultados e Discussão}

A área de estudo pode ser caracterizada como um remanescente de Floresta Estacional Semidecidual (FES) por ocorrer entre a área costeira-úmida e interiorana-árida, pelo baixo número de espécies epífitas (ca. 1\%) e pela estação seca bem marcada, com índices pluviométricos entre 62 a $87 \mathrm{~mm}$ mensais no período seco e 118 a $175 \mathrm{~mm}$ no período chuvoso (Embrapa 2014).

Foram registradas 192 espécies, distribuídas em 139 gêneros e 59 famílias (tabela 1 , figuras 4, 5). As famílias mais representativas foram Fabaceae (18 spp.), Rubiaceae (15 spp.), Asteraceae (12 spp.), Bignoniaceae, Myrtaceae e Sapindaceae (10 spp. cada) e Malvaceae (nove spp.) (figura 2). Das espécies amostradas, 76 espécies (39\%) tem hábito arbóreo, com alturas média de $7 \mathrm{~m}$, e as emergentes entre 10 a $12 \mathrm{~m}$ de altura, 59 espécies (31\%) pertencem ao hábito arbustivo, 40 (21\%) são trepadeiras herbáceas ou lenhosas, $15(8 \%)$ são ervas, 2 (1\%) são epífitas (figura 3). Os gêneros mais representativos foram Solanum (cinco spp.), Cupania, Eugenia, Guapira e Myrcia (quatro spp. cada), Casearia, Coccoloba, Erythroxylum, Lantana, Miconia, Ocotea, Passiflora, Pavonia, Psychotria, Senna e Serjania (três spp. cada) (tabela 1).

Os levantamentos florísticos realizados em áreas no Domínio Atlântico no Nordeste do país (Amorim et al. 2005, Carvalho-Sobrinho \& Queiroz 2005, Rodal et al. 2005, Pereira \& Alves 2007, Amorim et al. 2008, entre outros), em sua maioria, destacaram as famílias Fabaceae, Myrtaceae e Rubiaceae entre as mais representativas, resultado semelhante ao encontrado neste estudo (tabela 2). Nota-se que as famílias Orchidaceae e Bromeliaceae estão entre

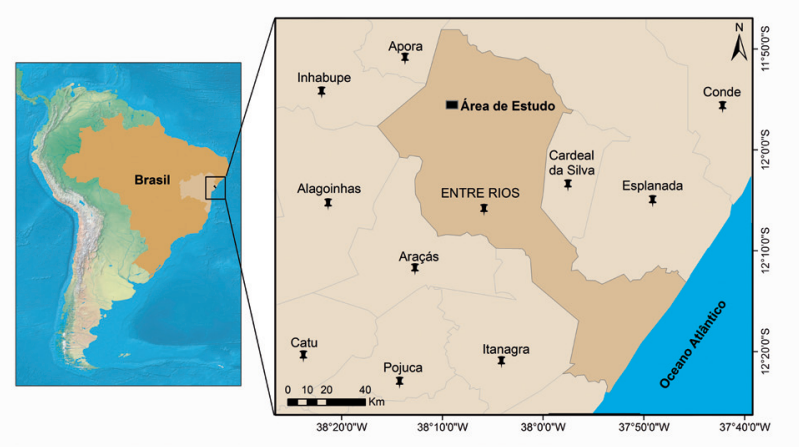

Figura 1. Município de Entre Rios, litoral norte do Estado da Bahia, Brasil, com destaque para a área de estudo.

Figure 1. Entre Rios municipality, northern coast of Bahia State, Brazil, with the area of study in detail. 
Tabela 1. Lista de famílias e espécies encontradas no remanescente de Mata Atlântica da FRC/UFBA, município de Entre Rios, BA, Brasil. *: Espécies endêmicas do Nordeste do Brasil; Não-DA: não citada para o Domínio Atlântico (Stehmann et al. 2009); Não-FES: não citada para a Floresta Estacional Semidecidual (Stehmann et al. 2009).

Table 1. List of families and species found in the remnant of Atlantic Forest in FRC/UFBA, municipality of Entre Rios, Bahia State, Brazil. *: Endemic species of the Northeast of Brazil; Não-DA: not cited for the Atlantic Domain (Stehmann et al. 2009); Não-FES: not cited for Semideciduous Forest (Stehmann et al. 2009.).

\begin{tabular}{|c|c|c|c|c|}
\hline Família/Espécies & Não-DA & Não-FES & Hábito & $\mathrm{N}^{\mathrm{o}} \mathrm{ALCB}$ \\
\hline \multicolumn{5}{|l|}{ Acanthaceae } \\
\hline Ruellia cearensis Lindau* & $\mathrm{X}$ & & Arbusto & 98035 \\
\hline \multicolumn{5}{|l|}{ Agavaceae } \\
\hline Herreria salsaparrilha Mart. & & & $\begin{array}{l}\text { Trepadeira } \\
\text { herbácea }\end{array}$ & 102627 \\
\hline \multicolumn{5}{|l|}{ Annonaceae } \\
\hline Annona salzmannii A.DC. & & $\mathrm{X}$ & Árvore & 93947 \\
\hline Duguetia furfuracea (A.St.-Hil.) Saff. & $\mathrm{X}$ & & Árvore & 94083 \\
\hline Guatteria sp. & & & Árvore & 94066 \\
\hline Xylopia laevigata Mart. & & $\mathrm{X}$ & Árvore & 90115 \\
\hline \multicolumn{5}{|l|}{ Apocynaceae } \\
\hline Aspidosperma spruceanum Benth. ex Mull. Arg. & & & Árvore & 101326 \\
\hline Himatanthus bracteatus (A.DC.) Woodson & & $\mathrm{X}$ & Árvore & 93940 \\
\hline Mandevilla moricandiana (A.DC.) Woodson & & $\mathrm{X}$ & $\begin{array}{l}\text { Trepadeira } \\
\text { herbácea }\end{array}$ & 94098 \\
\hline $\begin{array}{l}\text { Mandevilla scabra (Hoffmanns. ex Roem. } \\
\text { \& Schult.) K. Schum. }\end{array}$ & & & $\begin{array}{l}\text { Trepadeira } \\
\text { herbácea }\end{array}$ & 94101 \\
\hline Temnadenia violacea (Vell.) Miers & & & $\begin{array}{l}\text { Trepadeira } \\
\text { herbácea }\end{array}$ & 93903 \\
\hline \multicolumn{5}{|l|}{ Araceae } \\
\hline Anthurium longipes N.E. Br.* & & $\mathrm{X}$ & Erva & 93587 \\
\hline \multicolumn{5}{|l|}{ Arecaceae } \\
\hline Bactris sp. & & & Arborescente & 93956 \\
\hline Syagrus botryophora Mart. & & $\mathrm{X}$ & Arborescente & 94079 \\
\hline Syagrus coronata (Mart.) Becc. & & $\mathrm{X}$ & Arborescente & 93936 \\
\hline \multicolumn{5}{|l|}{ Asteraceae } \\
\hline Ageratum conyzoides L. & & $\mathrm{X}$ & Arbusto & 97239 \\
\hline Centratherum punctatum Cass. & & $\mathrm{X}$ & Arbusto & 93533 \\
\hline Conocliniopsis prasiifolia (DC.) R.M. Harley & & $\mathrm{X}$ & Arbusto & 90129 \\
\hline Dasyphyllum lanceolatum (Less.) Cabrera & & $\mathrm{X}$ & $\begin{array}{l}\text { Trepadeira } \\
\text { lenhosa }\end{array}$ & 97236 \\
\hline Eremanthus sp. & & & Árvore & 94784 \\
\hline Lepidaploa cotoneaster (Willd. ex Spreng.) H. Rob. & $\mathrm{X}$ & & Arbusto & 97238 \\
\hline Lepidaploa gracilis (Kunth) H. Rob. & $\mathrm{X}$ & & Arbusto & 90123 \\
\hline Mikania sp. & & & $\begin{array}{l}\text { Trepadeira } \\
\text { herbácea }\end{array}$ & 94110 \\
\hline Moquiniastrum oligocephalum (Gardner) G. Sancho & $\mathrm{X}$ & & Árvore & 90116 \\
\hline
\end{tabular}


Tabela 1 (continuação)

\begin{tabular}{|c|c|c|c|c|}
\hline Família/Espécies & Não-DA & Não-FES & Hábito & $\mathrm{N}^{\circ} \mathrm{ALCB}$ \\
\hline $\begin{array}{l}\text { Platypodanthera melissifolia (DC.) R.M. King } \\
\text { \& H. Rob. }\end{array}$ & $\mathrm{X}$ & & Arbusto & 90128 \\
\hline Tilesia baccata (L.) Pruski & & & Arbusto & 97240 \\
\hline Verbesina macrophylla (Cass.) S.F. Blake & & & Arbusto & 90099 \\
\hline \multicolumn{5}{|l|}{ Bignoniaceae } \\
\hline $\begin{array}{l}\text { Amphilophium scabriusculum (Mart. ex DC.) } \\
\text { L.G. Lohmann }\end{array}$ & & $X$ & $\begin{array}{l}\text { Trepadeira } \\
\text { herbácea }\end{array}$ & 97287 \\
\hline Bignonia corymbosa (Venth.) L.G. Lohmann & & $X$ & $\begin{array}{l}\text { Trepadeira } \\
\text { lenhosa }\end{array}$ & 94792 \\
\hline Fridericia platyphylla (Cham.) L.G. Lohmann & & & $\begin{array}{l}\text { Trepadeira } \\
\text { lenhosa }\end{array}$ & 94776 \\
\hline Fridericia sp. & & & $\begin{array}{l}\text { Trepadeira } \\
\text { lenhosa }\end{array}$ & 101708 \\
\hline Handroanthus impetiginosus Mattos & $\mathrm{X}$ & & Árvore & 101322 \\
\hline Handroanthus sp. & & & Árvore & 94080 \\
\hline Lundia corymbifera (Vahl) Sandwith & & $\mathrm{X}$ & $\begin{array}{l}\text { Trepadeira } \\
\text { lenhosa }\end{array}$ & 104744 \\
\hline Stizophyllum riparium (Kunth) Sandwith & & $\mathrm{X}$ & $\begin{array}{l}\text { Trepadeira } \\
\text { lenhosa }\end{array}$ & 94779 \\
\hline Tabebuia elliptica (DC.) Sandwith & & $\mathrm{X}$ & Árvore & 94081 \\
\hline Tabebuia stenocalyx Spreng. \& Stapf. & & $\mathrm{X}$ & Árvore & 93932 \\
\hline \multicolumn{5}{|l|}{ Boraginaceae } \\
\hline Cordia pilosa M. Stapf \& Taroda* & $\mathrm{X}$ & & Arbusto & 93582 \\
\hline Tournefortia gardneri A.DC. & & $\mathrm{X}$ & Arbusto & 90140 \\
\hline \multicolumn{5}{|l|}{ Bromeliaceae } \\
\hline Aechmea multiflora L.B. Smith & & & Erva/Epífita & 93942 \\
\hline \multicolumn{5}{|l|}{ Calophyllaceae } \\
\hline Kielmeyera neglecta Saddi* & & $\mathrm{X}$ & Árvore & 94087 \\
\hline \multicolumn{5}{|l|}{ Celastraceae } \\
\hline Hippocratea volubilis L. & & & $\begin{array}{l}\text { Trepadeira } \\
\text { lenhosa }\end{array}$ & 94102 \\
\hline Maytenus distichophylla Mart. ex Reissek & & $X$ & Árvore & 94074 \\
\hline Maytenus obtusifolia Mart. & & $X$ & Árvore & 90147 \\
\hline \multicolumn{5}{|l|}{ Chrysobalanaceae } \\
\hline Hirtella racemosa Lam. & & $X$ & Árvore & 93910 \\
\hline \multicolumn{5}{|l|}{ Clusiaceae } \\
\hline Clusia nemorosa G. Mey. & & $\mathrm{X}$ & Árvore & 93915 \\
\hline \multicolumn{5}{|l|}{ Connaraceae } \\
\hline Rourea gardneriana Planch. & & $X$ & Arbusto & 93923 \\
\hline \multicolumn{5}{|l|}{ Convolvulaceae } \\
\hline Jacquemontia glaucescens Choisy & & $\mathrm{X}$ & $\begin{array}{l}\text { Trepadeira } \\
\text { herbácea }\end{array}$ & 90103 \\
\hline
\end{tabular}


Tabela 1 (continuação)

$\frac{\text { Família/Espécies }}{\text { Jacquemontia pentanthos (Jacq.) G. Don }}$

Não-DA

Cyperaceae

Rhynchospora exaltata Kunth

Scleria bracteata Cav.

Dilleniaceae

Davilla nitida (Vahl) Kubitzki

Davilla sessilifolia Fraga*

Tetracera boomii Aymard

Erythroxylaceae

Erythroxylum mikanii Peyr

Erythroxylum nobile O.E. Schulz*

Erythroxylum passerinum Mart.*

Euphorbiaceae

Croton heliotropiifolius Kunth

Croton sellowii Baill.*

Dalechampia ilheotica Wawra

Dalechampia peckoltiana Müll. Arg.

Tragia volubilis L.

Fabaceae

Abarema cochliacarpos (F.S. Gomes) Barneby \& J.W. Grimes

Albizia polycephala (Benth.) Killip ex Record

Apuleia leiocarpa (Vog.) J.F. Macbr.

Bauhinia sp. nov.ined.*

Chamaecrista swainsonii (Benth.) H.S. Irwin \& Barneby*

Dioclea lasiophylla Mart. ex Benth.*

Mimosa caesalpiniifolia Benth.

Periandra mediterranea (Vell.) Taub.

Piptadenia ramosissima Benth.*

Poecilanthe itapuana G.P. Lewis*

Rhynchosia phaseoloides (Sw.) DC.
X

$\begin{array}{ccc}\text { Não-FES } & \text { Hábito } & \mathrm{N}^{\circ} \text { ALCB } \\ & \begin{array}{c}\text { Trepadeira } \\ \text { herbácea }\end{array} & 94095 \\ \text { X } & \text { Erva } & 94783 \\ \text { X } & \text { Erva } & 94200\end{array}$

X

Trepadeira
lenhosa

94769

Trepadeira lenhosa

X Trepadeira

lenhosa

94771

93920

X Arbusto

94104

$\mathrm{X}$

Arbusto

94103

X Árvore

101311

X

Arbusto

93526

$\mathrm{X}$

Arbusto

93931

$\mathrm{X}$

Trepadeira

herbácea

93537

Trepadeira

herbácea

93574

Trepadeira

herbácea

93567

Árvore

93525

Árvore

97285

Árvore

93937

Arbusto

90104

Arbusto

94109

Trepadeira

herbácea

93564

Árvore

90131

$\mathrm{X}$

Arbusto

93591

Trepadeira

lenhosa

Árvore

97284

X

Trepadeira

herbácea
101319

90152 
Tabela 1 (continuação)

\begin{tabular}{|c|c|c|c|c|}
\hline Família/Espécies & Não-DA & Não-FES & Hábito & $\mathrm{N}^{\mathrm{o}} \mathrm{ALCB}$ \\
\hline $\begin{array}{l}\text { Senna macranthera (DC. ex Collad.) H.S. Irwin } \\
\text { \& Barneby }\end{array}$ & & & Árvore & 93908 \\
\hline Senna obtusifolia (L.) H.S. Irwin \& Barneby & & & Arbusto & 93535 \\
\hline Senna phlebadenia H.S. Irwin \& Barneby* & & $X$ & Árvore & 93916 \\
\hline Stryphnodendron pulcherrimum (Willd.) Hochr. & & & Árvore & 90143 \\
\hline Stylosanthes guianensis (Aubl.) Sw. & & & Erva & 94202 \\
\hline Swartzia apetala Raddi & & & Árvore & 93913 \\
\hline $\begin{array}{l}\text { Tachigali densiflora (Benth.) L.G. Silva \& } \\
\text { H.C. Lima }\end{array}$ & & & Árvore & 101297 \\
\hline \multicolumn{5}{|l|}{ Gentianaceae } \\
\hline $\begin{array}{l}\text { Chelonanthus purpurascens (Aubl.) Struwe, } \\
\text { S. Nilsson \& V.A. Albert }\end{array}$ & & $\mathrm{X}$ & Erva & 97481 \\
\hline Coutoubea spicata Aubl. & & $\mathrm{X}$ & Erva & 93899 \\
\hline \multicolumn{5}{|l|}{ Hernandiaceae } \\
\hline Sparattanthelium botocudoru Mart. & & & Arbusto & 94092 \\
\hline \multicolumn{5}{|l|}{ Hypericaceae } \\
\hline Vismia guianensis (Aubl.) Choisy & $X$ & & Arbusto & 94112 \\
\hline \multicolumn{5}{|l|}{ Iridaceae } \\
\hline Trimezia martinicensis (Jacq.) Herb. & & & Erva & 90102 \\
\hline \multicolumn{5}{|l|}{ Krameriaceae } \\
\hline Krameria tomentosa A. St-Hil. & & $\mathrm{X}$ & Arbusto & 94786 \\
\hline \multicolumn{5}{|l|}{ Lamiaceae } \\
\hline $\begin{array}{l}\text { Eplingiella fruticosa (Salzm. ex Benth.) Harley \& } \\
\text { J.F.B. Pastore* }\end{array}$ & & $\mathrm{X}$ & Arbusto & 94791 \\
\hline \multicolumn{5}{|l|}{ Lauraceae } \\
\hline Ocotea canaliculata (Rich.) Mez. & $\mathrm{X}$ & & Árvore & 101296 \\
\hline Ocotea glomerata (Ness) Mez. & $X$ & & Árvore & 93579 \\
\hline Ocotea notata (Nees \& Mart.) Mez. & & & Árvore & 94069 \\
\hline \multicolumn{5}{|l|}{ Lecythidaceae } \\
\hline Eschweilera ovata (Cambess.) Mart. ex Miers & & $\mathrm{X}$ & Árvore & 93623 \\
\hline \multicolumn{5}{|l|}{ Loganiaceae } \\
\hline Strychnos brasiliensis (Spreng.) Mart. & & $\mathrm{X}$ & $\begin{array}{l}\text { Trepadeira } \\
\text { lenhosa }\end{array}$ & 102624 \\
\hline \multicolumn{5}{|l|}{ Malpighiaceae } \\
\hline Byrsonima sericea DC. & & X & Árvore & 93935 \\
\hline $\begin{array}{l}\text { Diplopterys pubipetala (A. Juss.) W.R. Anderson } \\
\text { \& C.C. Davis }\end{array}$ & $\mathrm{X}$ & & $\begin{array}{l}\text { Trepadeira } \\
\text { lenhosa }\end{array}$ & 93513 \\
\hline Janusia anisandra (A. Juss.) Griseb. & & $\mathrm{X}$ & $\begin{array}{l}\text { Trepadeira } \\
\text { herbácea }\end{array}$ & 94788 \\
\hline \multicolumn{5}{|l|}{ Malvaceae } \\
\hline Eriotheca sp. & & & Árvore & 93949 \\
\hline
\end{tabular}


Tabela 1 (continuação)

\begin{tabular}{|c|c|c|c|c|}
\hline Família/Espécies & Não-DA & Não-FES & Hábito & $\mathrm{N}^{\mathrm{o}} \mathrm{ALCB}$ \\
\hline Helicteres laciniosa Cristóbol* & & $\mathrm{X}$ & Arbusto & 90107 \\
\hline Luehea paniculata Mart. \& Zucc. & & & Árvore & 93941 \\
\hline Melochia tomentosa $\mathrm{L}$. & & & Arbusto & 94196 \\
\hline Pavonia malacophylla (Link \& Otto) Garcke & & & Arbusto & 97480 \\
\hline Pavonia martii Colle. & & & Arbusto & 94114 \\
\hline Pavonia sidifolia Kunth & & & Arbusto & 94201 \\
\hline Sida linifolia Cav. & & $\mathrm{X}$ & Erva & 90120 \\
\hline Sida ulei Ulbr. & $\mathrm{X}$ & & Arbusto & 93545 \\
\hline \multicolumn{5}{|l|}{ Melastomataceae } \\
\hline Miconia albicans (Sw.) A. Triana & & & Árvore & 93597 \\
\hline Miconia amoena A. Triana & & $X$ & Arbusto & 93615 \\
\hline Miconia ciliata (L.C. Rich.) DC. & & $\mathrm{X}$ & Arbusto & 93578 \\
\hline \multicolumn{5}{|l|}{ Meliaceae } \\
\hline Trichilia lepidota Mart. & & & Árvore & 93926 \\
\hline \multicolumn{5}{|l|}{ Moraceae } \\
\hline Ficus hirsuta Schott & $\mathrm{X}$ & & Árvore & 94067 \\
\hline \multicolumn{5}{|l|}{ Myrtaceae } \\
\hline Calycolpus legrandii Mattos* & & $\mathrm{X}$ & Árvore & 93909 \\
\hline Eugenia astringens Cambess. & $\mathrm{X}$ & & Árvore & 101306 \\
\hline Eugenia ligustrina (Sw.) Willd. & & & Arbusto & 94790 \\
\hline Eugenia sp.1 & & & Árvore & 101306 \\
\hline Eugenia sp. 2 & & & Arbusto & 90112 \\
\hline Myrcia decorticans DC. & & & Árvore & 93939 \\
\hline Myrcia guianensis (Aubl.) DC. & & & Árvore & 94068 \\
\hline Myrcia rosangelae NicLugh.* & $\mathrm{X}$ & & Árvore & 94111 \\
\hline Myrcia splendens (SW.) DC. & & & Árvore & 101303 \\
\hline Plinia cauliflora (Mart.) Kausel & & & Árvore & 93945 \\
\hline \multicolumn{5}{|l|}{ Nyctaginaceae } \\
\hline Guapira graciliflora (Mart. ex Schmidt) Lundell & $\mathrm{X}$ & & Árvore & 94084 \\
\hline Guapira hirsuta (Choisy) Lundell & & & Árvore & 101308 \\
\hline Guapira obtusata (Jacq.) Litlle & & $\mathrm{X}$ & Árvore & 94078 \\
\hline Guapira opposita (Vell.) Reitz & & & Árvore & 101314 \\
\hline \multicolumn{5}{|l|}{ Ochnaceae } \\
\hline Ouratea cuspidata (A.St.-Hil.) Engl. & & $\mathrm{X}$ & Árvore & 101299 \\
\hline Sauvagesia erecta $\mathrm{L}$. & & $\mathrm{X}$ & Erva & 94198 \\
\hline \multicolumn{5}{|l|}{ Olacaceae } \\
\hline Ximenia americana $\mathrm{L}$. & & $\mathrm{X}$ & Árvore & 101317 \\
\hline \multicolumn{5}{|l|}{ Orchidaceae } \\
\hline $\begin{array}{l}\text { Koellensteinia spiralis Gomes-Ferreira } \\
\text { \& L.C. Menezes* }\end{array}$ & & $\mathrm{X}$ & Erva & 94773 \\
\hline
\end{tabular}


Tabela 1 (continuação)

\begin{tabular}{|c|c|c|c|c|}
\hline Família/Espécies & Não-DA & Não-FES & Hábito & $\mathrm{N}^{\circ}$ ALCB \\
\hline Notylia microchila Cogn. & $\mathrm{X}$ & & Erva & 98048 \\
\hline Sacoila lanceolata (Aubl.) Garay & & $\mathrm{X}$ & Erva & 98047 \\
\hline Specklinia ianthina E. Pessoa \& F. Barros* & $\mathrm{X}$ & & Epífita & 98049 (Parátipo) \\
\hline Vanilla bahiana Hoehne & & $\mathrm{X}$ & $\begin{array}{l}\text { Trepadeira } \\
\text { herbácea }\end{array}$ & 94094 \\
\hline \multicolumn{5}{|l|}{ Passifloraceae } \\
\hline Passiflora cincinnata Mast. & & & $\begin{array}{l}\text { Trepadeira } \\
\text { herbácea }\end{array}$ & 102625 \\
\hline Passiflora foetida L. & & $\mathrm{X}$ & $\begin{array}{l}\text { Trepadeira } \\
\text { herbácea }\end{array}$ & 93654 \\
\hline Passiflora mucronata Lam. & & $\mathrm{X}$ & $\begin{array}{l}\text { Trepadeira } \\
\text { herbácea }\end{array}$ & 94097 \\
\hline \multicolumn{5}{|l|}{ Phyllanthaceae } \\
\hline Margaritaria nobilis L.f. & & & Árvore & 101315 \\
\hline \multicolumn{5}{|l|}{ Plantaginaceae } \\
\hline Scoparia dulcis L. & & & Arbusto & 93289 \\
\hline Stemodia foliosa Benth. & & $\mathrm{X}$ & Arbusto & 94197 \\
\hline \multicolumn{5}{|l|}{ Polygalaceae } \\
\hline $\begin{array}{l}\text { Asemeia violacea (Aubl.) J.F.B. Pastore \& } \\
\text { J.R. Abbott }\end{array}$ & & $\mathrm{X}$ & Erva & 93553 \\
\hline Polygala paniculata $\mathrm{L}$. & & & Erva & 93570 \\
\hline \multicolumn{5}{|l|}{ Polygonaceae } \\
\hline Coccoloba lucidula Benth. & & & $\begin{array}{l}\text { Trepadeira } \\
\text { lenhosa }\end{array}$ & 94772 \\
\hline Coccoloba parimensis Benth. & & & $\begin{array}{l}\text { Trepadeira } \\
\text { lenhosa }\end{array}$ & 90154 \\
\hline Coccoloba ramosissima Wedd. & & $\mathrm{X}$ & Árvore & 101335 \\
\hline \multicolumn{5}{|l|}{ Proteaceae } \\
\hline Roupala montana Aubl. & & & Árvore & 101394 \\
\hline \multicolumn{5}{|l|}{ Rhamnaceae } \\
\hline Ziziphus cf. platyphylla Reissek & & & Árvore & 101328 \\
\hline \multicolumn{5}{|l|}{ Rubiaceae } \\
\hline Alseis floribunda Schott. & & $\mathrm{X}$ & Árvore & 93962 \\
\hline Alseis pickelii Pilg. \& Schmale* & & & Árvore & 93944 \\
\hline Amaioua guianensis Aubl. & & $\mathrm{X}$ & Arbusto & 98044 \\
\hline Cordiera macrophylla (K. Schum.) Kuntze & & $\mathrm{X}$ & Arbusto & 93620 \\
\hline Cordiera obtusa (K. Schum.) Kuntze & & $\mathrm{X}$ & Arbusto & 93612 \\
\hline Coutarea hexandra (Jacq.) K. Schum. & & & Árvore & 94085 \\
\hline $\begin{array}{l}\text { Denscantia monodon (K. Schum.) E.L. Cabral } \\
\text { \& Bacigalupo* }\end{array}$ & & $\mathrm{X}$ & $\begin{array}{l}\text { Trepadeira } \\
\text { herbácea }\end{array}$ & 90110 \\
\hline Guettarda viburnoides Cham. \& Schltdl. & & & Árvore & 90111 \\
\hline
\end{tabular}


Tabela 1 (continuação)

\begin{tabular}{|c|c|c|c|c|}
\hline Família/Espécies & Não-DA & Não-FES & Hábito & $\mathrm{N}^{\circ} \mathrm{ALCB}$ \\
\hline Leptoscela ruellioides Hook. f.* & $\mathrm{X}$ & & Arbusto & 94768 \\
\hline Malanea evenosa Müll. Arg. & & $\mathrm{X}$ & Arbusto & $93656 a$ \\
\hline Margaritopsis chaenotricha (DC.) C.M. Taylor & & & Arbusto & 93560 \\
\hline Psychotria bracteocardia (DC.) Müll. Arg. & & & Arbusto & 90132 \\
\hline Psychotria capitata Ruiz \& Pavon & & & Arbusto & 94105 \\
\hline $\begin{array}{l}\text { Psychotria hoffmannseggiana (Willd. ex } \\
\text { Schult.) Müll. Arg. }\end{array}$ & & & Arbusto & 90117 \\
\hline Salzmannia nitida DC.* & & & Arbusto & 93585 \\
\hline \multicolumn{5}{|l|}{ Rutaceae } \\
\hline Zanthoxylum monogynum A.St.-Hil. & & & Árvore & 93562 \\
\hline \multicolumn{5}{|l|}{ Salicaceae } \\
\hline Banara parviflora (A. Gray.) Benth. & & $X$ & Árvore & 101321 \\
\hline Casearia arborea (L.C. Ricch.) Urban & & $\mathrm{X}$ & Árvore & 93596 \\
\hline Casearia bahiensis Sleumer & & $\mathrm{X}$ & Árvore & 94075 \\
\hline Casearia commersoniana Cambess. & & $\mathrm{X}$ & Árvore & 94073 \\
\hline \multicolumn{5}{|l|}{ Santalaceae } \\
\hline Phoradendron strongyloclados Eichl. & & $X$ & $\begin{array}{c}\text { Erva } \\
\text { hemiparasita }\end{array}$ & 94785 \\
\hline \multicolumn{5}{|l|}{ Sapindaceae } \\
\hline Allophylus edulis (A.St.-Hil.) Radlk. & & & Árvore & 94077 \\
\hline Cupania bracteosa Radlk. & & $\mathrm{X}$ & Arbusto & 93952 \\
\hline Cupania racemosa (Vell.) Radlk. & & & Árvore & 93914 \\
\hline Cupania rigida Radlk. & & & Árvore & 93554 \\
\hline Cupania rugosa Radlk. & & & Arbusto & 90171 \\
\hline Melicoccus oliviformis Kunth & & $\mathrm{X}$ & Árvore & 94072 \\
\hline Paullinia racemosa Waura & & $\mathrm{X}$ & $\begin{array}{l}\text { Trepadeira } \\
\text { lenhosa }\end{array}$ & 93954 \\
\hline Serjania clematidifolia Cambess. & & $\mathrm{X}$ & $\begin{array}{l}\text { Trepadeira } \\
\text { herbácea }\end{array}$ & 90148 \\
\hline Serjania paradoxa Radlk. & & & $\begin{array}{l}\text { Trepadeira } \\
\text { herbácea }\end{array}$ & 93552 \\
\hline Serjania salzmanniana Schltdl. & & & $\begin{array}{l}\text { Trepadeira } \\
\text { herbácea }\end{array}$ & 90137 \\
\hline \multicolumn{5}{|l|}{ Sapotaceae } \\
\hline Chrysophyllum rufum Mart. & & $\mathrm{X}$ & Àrvore & 90098 \\
\hline Pouteria gardneri (Mart. \& Miq.) Baehni & & & Árvore & 101320 \\
\hline Pouteria sp. & & & Árvore & 101298 \\
\hline \multicolumn{5}{|l|}{ Schoepfiaceae } \\
\hline Schoepfia brasiliensis A.DC. & & & Árvore & 94076 \\
\hline \multicolumn{5}{|l|}{ Smilacaceae } \\
\hline Smilax rufescens Griseb. & $\mathrm{X}$ & & $\begin{array}{l}\text { Trepadeira } \\
\text { herbácea }\end{array}$ & 93602 \\
\hline
\end{tabular}


Tabela 1 (continuação)

\begin{tabular}{|c|c|c|c|c|}
\hline Família/Espécies & Não-DA & Não-FES & Hábito & $\mathrm{N}^{\circ} \mathrm{ALCB}$ \\
\hline Smilax sp. & & & $\begin{array}{l}\text { Trepadeira } \\
\text { herbácea }\end{array}$ & 94777 \\
\hline \multicolumn{5}{|l|}{ Solanaceae } \\
\hline Solanum asperum L.C. Rich. & & & Arbusto & 93516 \\
\hline Solanum mauritianum Scop. & & & Arbusto & 93517 \\
\hline Solanum paludosum Moric. & & $X$ & Arbusto & 90138 \\
\hline Solanum polytrichum Moric. & & $\mathrm{X}$ & Arbusto & 93520 \\
\hline Solanum rupincola Sendt. & & $\mathrm{X}$ & Arbusto & 90109 \\
\hline \multicolumn{5}{|l|}{ Trigoniaceae } \\
\hline Trigonia nivea Cabess. & & $\mathrm{X}$ & Arbusto & 93624 \\
\hline \multicolumn{5}{|l|}{ Turneraceae } \\
\hline Turnera calyptrocarpa Urb. & & & Arbusto & 94108 \\
\hline \multicolumn{5}{|l|}{ Verbenaceae } \\
\hline Lantana fucata Lindl. & & $\mathrm{X}$ & Arbusto & 90126 \\
\hline Lantana radula $\mathrm{Sw}$. & $\mathrm{X}$ & & $\begin{array}{l}\text { Trepadeira } \\
\text { lenhosa }\end{array}$ & 94787 \\
\hline Lantana undulata Scrank & & $\mathrm{X}$ & Arbusto & 90106 \\
\hline Lippia macrophylla Cham.* & $\mathrm{X}$ & & Arbusto & 93534 \\
\hline
\end{tabular}

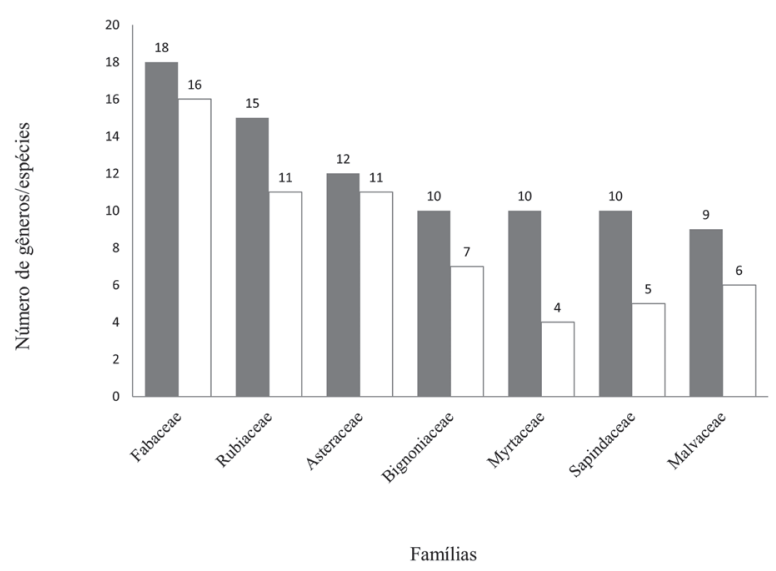

Figura 2. Número de gêneros e espécies das famílias mais representativas encontradas no remanescente da FRC/UFBA.

Figure 2. Number of genera and species of the most representative families found in the remnant of FRC /UFBA.

as mais representativas em Florestas Ombrófilas, montanas ou em áreas transicionais (Amorim et al. 2005, Carvalho Sobrinho \& Queiroz 2005, Amorim et al. 2008), enquanto Poaceae e Cyperaceae estão bem representadas nos levantamentos em áreas de restinga (Oliveira Filho \& Carvalho 1993, Almeida Jr. et al. 2007, Mendes et al. 2010, Queiroz et al. 2012, Silva \& Menezes 2012).

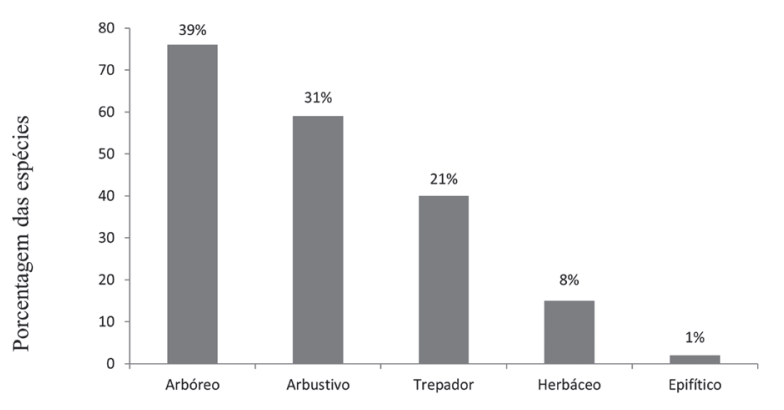

Figura 3. Hábito das espécies encontradas no remanescente da FRC/UFBA.

Figure 3. Habit of the species found in the remnant of FRC/UFBA.

Quando comparado aos trabalhos citados anteriormente, a Serra da Itabaiana em Sergipe (Mendes et al. 2010) destacou-se pelo maior número de espécies em comum (58) com o remanescente da FRC/UFBA. Destas, nove espécies foram registradas somente para estas duas localidades (Chamaecrista swainsonii (Benth.) H.S. Irwin \& Barneby, Lantana radula Sw., Margaritopsis chaenotricha (DC.) 
C.M. Taylor, Maytenus obtusifolia Mart., Tetracera boomii Aymard, Trimezia martinicensis (Jacq.) Herb., Mandevilla moricandiana (A.DC.) Woodson, Coccoloba lucidula Benth., Jacquemontia pentanthos (Jacq.) G. Don). Esta similaridade na flora ocorreu, provavelmente, devido à proximidade geográfica entre as áreas e ao fato de a Serra da Itabaiana ser uma área de tensão ecológica entre Caatinga e Mata Atlântica, possuindo, desta forma, uma grande quantidade de espécies comuns à Florestas Estacionais Semideciduais do Domínio Atlântico.

Destaca-se, também, a grande quantidade de espécies com hábito trepador (21\%) encontradas no remanescente da FRC/UFBA, quando comparado a outros inventários no Nordeste, onde as trepadeiras (lenhosas ou não) ocorreram em até $12 \%$ das espécies (Pereira \& Alves 2007, Amorim et al. 2008, Queiroz et al. 2012). Além de a elevada riqueza de trepadeiras

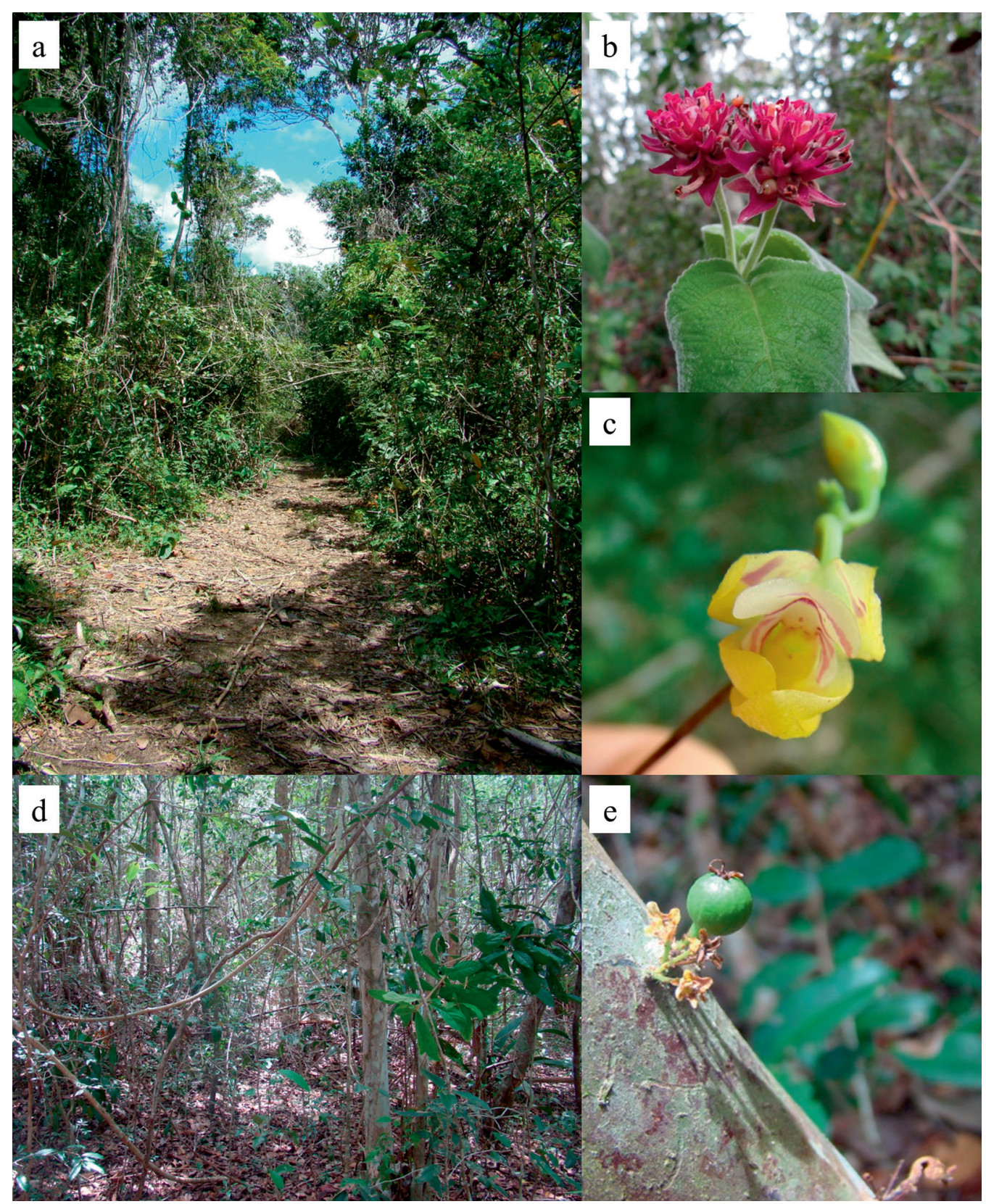

Figura 4. Remanescente de Mata Atlântica na FRC/UFBA, município de Entre Rios, BA, Brasil. a. Trilha pré-existente. b. Lippia macrophylla. c. Koellensteinia spiralis. d. subosque. e. Plinia cauliflora. Fotos: Roque, N.

Figure 4. Remnant of Atlantic Forest in FRC/UFBA, municipality of Entre Rios, Bahia State, Brazil. a. Trail. b. Lippia macrophylla. c. Koellensteinia spiralis. d. understory. e. Plinia cauliflora. Photos: Roque, N. 
ser um importante atributo de fragmentos de Florestas Estacionais Semideciduais (Udulutsch et al. 2004), o presente estudo concentrou esforços de coleta neste hábito.

Das espécies encontradas, 25 (13\%) têm distribuição restrita ao Nordeste do Brasil (tabela 1) e destas, dez possuem registro apenas para a Bahia (Anthurium longipes N.E. Br., Davilla sessilifolia Fraga, Denscantia monodon (K. Schum.) E.L. Cabral \& Bacigalupo, Helicteres laciniosa Cristóbol,
Kielmeyera neglecta Saddi, Koellensteinia spiralis Gomes-Ferreira \& L.C. Menezes, Lippia macrophylla Cham., Piptadenia ramosissima Benth., Poecilanthe itapuana G.P. Lewis, Specklinia ianthina E. Pessoa \& F. Barros).

Dentre as espécies coletadas no remanescente da FRC/UFBA, dois gêneros (Descantia, Salzmannia) e $35(18 \%)$ espécies são considerados endêmicos do Domínio Atlântico (Stehmann et al. 2009). Das espécies encontradas na área de estudo, 13\% não

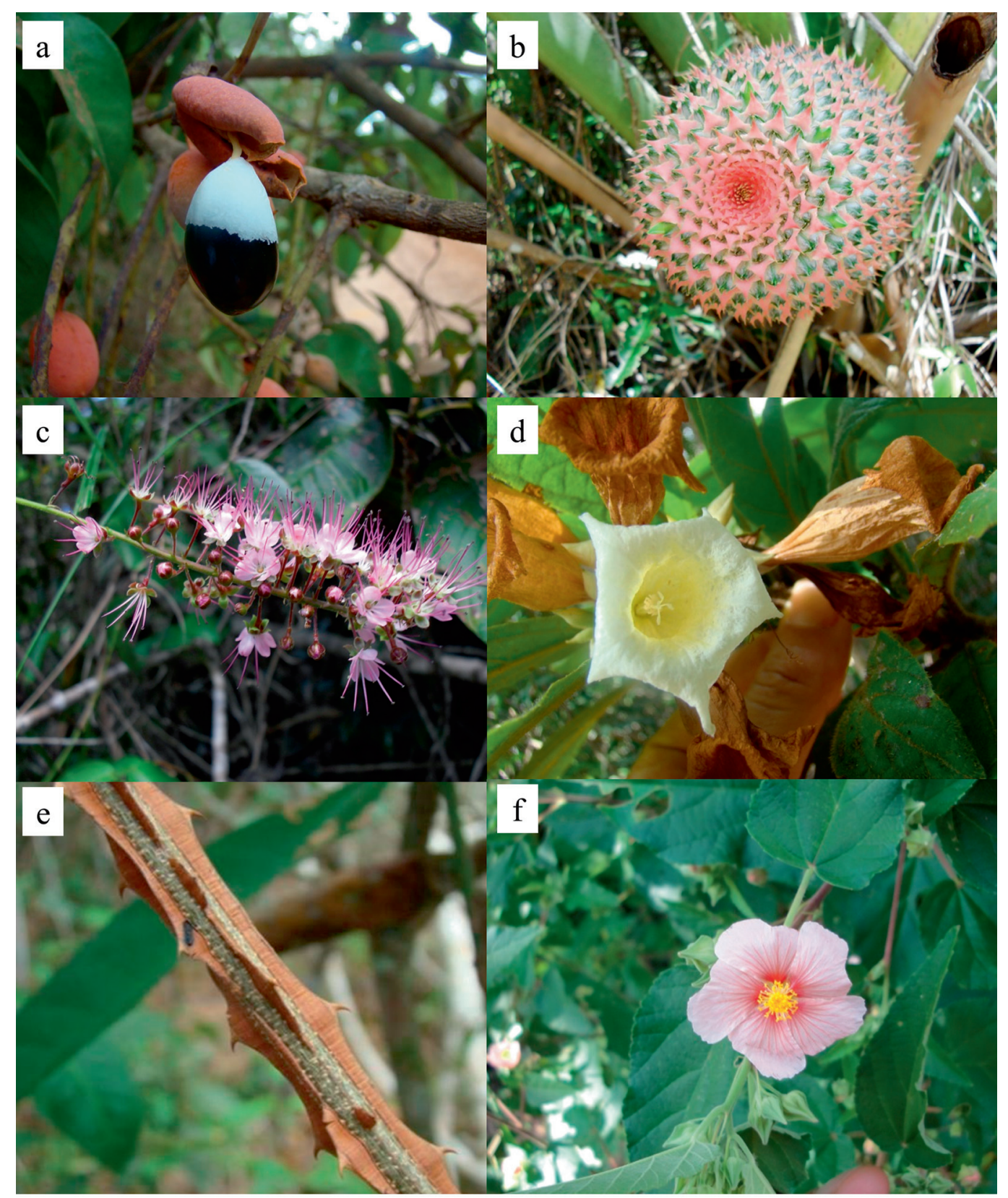

Figura 5. a. Swartzia apetala. b. Aechmea multiflora. c. Hirtella racemosa. d. Cordia pilosa. e. Piptadenia ramosissima. f. Pavonia malacophylla. Fotos: Roque, N.

Figure 5. a. Swartzia apetala. b. Aechmea multiflora. c. Hirtella racemosa. d. Cordia pilosa. e. Piptadenia ramosissima. f. Pavonia malacophylla. Photos: Roque, N. 


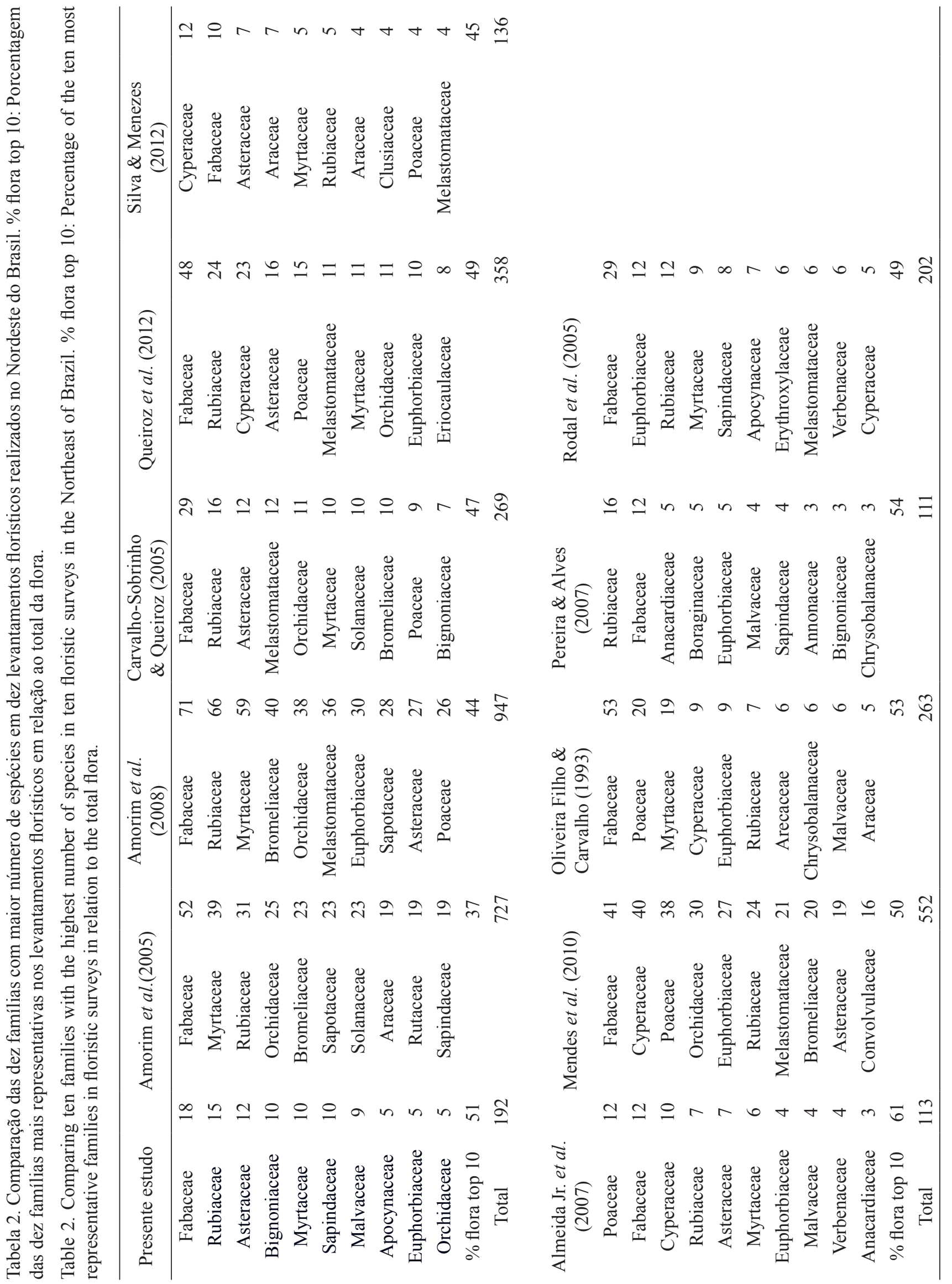


foram citadas por Stehmann et al. (2009) para este Domínio e $45 \%$ foram citadas para outras fisionomias, exceto Florestas Estacionais Semideciduais (tabela 1).

Foi registrada a ocorrência de duas espécies novas para a ciência, Bauhinia sp. nov. (L.P. de Queiroz, com. pess.), encontrada em Entre Rios e em outros municípios do litoral norte baiano e em Sergipe, e Specklinia ianthina E. Pessoa \& F. Barros, epífita com até $2 \mathrm{~cm}$ de altura com registro apenas dos materiais-tipo, três no município de Jequié e um na área do presente trabalho (Pessoa et al. 2014). Cordia pilosa M. Stapf \& Taroda, Davilla sessilifolia Fraga e Tetracera boomii Aymard têm o município de Entre Rios como localidade típica.

Apuleia leiocarpa (Vog.) J.F. Macbr., e Calycolpus legrandii Mattos foram classificadas como espécies vulneráveis (VU) (Martinelli \& Moraes 2013). Segundo estes autores, A. leiocarpa, apesar de amplamente distribuída no Brasil, tem sido muito utilizada pela indústria madeireira, e C. legrandii, por ocorrer em populações restritas nos Estados de Alagoas, Bahia e Sergipe, principalmente na restinga, áreas que vêm sofrendo forte degradação pela especulação imobiliária.

Outras espécies merecem atenção por possuírem menos de dez registros de coleta, entre elas, Piptadenia ramosissima Benth. e Koellensteinia spiralis GomesFerreira \& L.C. Menezes. Destacam-se, também, Cordia pilosa M. Stapf \& Taroda, classificada como espécie Deficiente em Dados (DD) e de ocorrência em vegetações de restinga nos Estados de Alagoas, Bahia e Sergipe (Stapf et al. 2010), além de Davilla sessilifolia Fraga, considerada rara e endêmica do Norte do Recôncavo Baiano (Fraga 2009).

Kasecker et al. (2009) definiram áreas-chave como sítios de significância global para a conservação da biodiversidade, afirmando que as mesmas podem ser baseadas em vulnerabilidade e insubstituibilidade, critérios comumente utilizados em planejamento sistemático da conservação. Os mesmos autores apontaram dois locais próximos a Entre Rios (município de Esplanada e a Fazenda do Bu, município de Conde) como áreas-chave, devido à presença de Davilla sessilifolia Fraga. A área do presente estudo, além de possuir a espécie citada, possui ainda quatro espécies com distribuição restrita, Cordia pilosa M. Stapf \& Taroda, Koellensteinia spiralis GomesFerreira \& L.C. Menezes, Piptadenia ramosissima Benth. e Specklinia ianthina E. Pessoa \& F. Barros, duas outras classificadas como vulneráveis, Apuleia leiocarpa (Vog.) J.F. Macbr., e Calycolpus legrandii
Mattos (Martinelli \& Moraes 2013), além de ser localidade tipo de Specklinia ianthina E. Pessoa \& F. Barros, podendo deste modo no futuro também ser considerada uma área-chave para a conservação da biodiversidade e vir a compor o Corredor da Mata Atlântica do Nordeste.

\section{Agradecimentos}

Os autores agradecem à Universidade Federal da Bahia pelo alojamento e transporte para a realização deste trabalho; aos taxonomistas que visitaram a coleção do Herbário ALCB e auxiliaram nas identificações; a Lídia Campos Nascimento pela confecção do mapa; aos revisores pelas sugestões ao manuscrito; às agências de fomento CNPq e FAPESB pelas bolsas de Iniciação Científica concedidas ao segundo autor e à terceira autora; aos projetos financiadores REFLORA (Proc. 563541/2010-5), PRONEM (PNE 1642/2011), INCT/HVFF e SiB-br/ $\mathrm{CNPq}$ e ao $\mathrm{CNPq}$ pela bolsa $\mathrm{PQ}$ concedida à última autora.

\section{Literatura citada}

Almeida Jr., E.B., Pimentel, R.M.M. \& Zickel, C.S. 2007. Flora e formas de vida em uma área de restinga no Litoral Norte de Pernambuco, Brasil. Revista de Geografia 24: 19-34.

Amane (Associação para a Proteção da Mata Atlântica do Nordeste). 2014. Mapa do Corredor da Mata Atlântica do Nordeste. Disponível em http://www. amane.org.br/publicacoes.asp?pag=2/ (acesso em 01-XII-2014).

Amorim, A.M., Fiaschi, P., Jardim, J.G., Thomas, W.W., Clifton, B.C. \& Carvalho, A.M.V. 2005. The vascular plants of a forest fragment in southern Bahia, Brazil. Sida 21: 1726-1752.

Amorim, A.M., Jardim, J.G., Lopes, M.M.M., Fiaschi, P., Borges, R.A.X., Perdiz, R.O. \& Thomas, W.W. 2009. Angiospermas em remanescentes de floresta montana no sul da Bahia, Brasil. Biota Neotropica 9: 313-348.

Amorim, A.M., Thomas, W.W., Carvalho, A.M.V. \& Jardim, J.G. 2008. Floristics of the Una Biological Reserve, Bahia, Brazil. In: W.W. Thomas (ed.). The Atlantic Coastal Forest of Northeastern Brazil. Memoirs of The New York Botanical Garden, New York, pp. 67-146.

APG III. 2009. An update of the Angiosperm Phylogeny Group classification for the orders and families of flowering plants: APG III. Botanical Journal of the Linnean Society 161: 105-121. 
Carvalho-Sobrinho, J.G. \& Queiroz, L.P. 2005. Composição Florística de um fragmento de Mata Atlântica na Serra da Jibóia, Santa Terezinha, Bahia, Brasil. Sitientibus série Ciências Biológicas 5: 20-28.

Coelho, M.M. \& Amorim, A.M. 2014. Floristic composition of the Montane Forest in the Almadina - Barro Preto axis, Southern Bahia, Brazil. Biota Neotropica 14: 1-41.

Embrapa. 2014. Banco de dados climáticos do Brasil. Disponível em http://www.bdclima.cnpm.embrapa.br/ (acesso em 01-XII-2014).

ESRI (Environmental Systems Reserch Institute, Inc.). 2010. ArcGIS version 9.3.1. Environmental Systems Reserch Institute, Inc. (United States of America).

Fraga, C.N. 2009. Dilleniaceae. In: Giulietti, A.M., Rapini, A., Andrade, M.J.G., Queiroz, L.P. \& Silva, J.M.C. (eds.). Plantas Raras do Brasil. Conservação Internacional, Belo Horizonte, pp. 159-160.

Gomes, F.S. \& Guedes, M.L.S. 2014. Flora vascular e formas de vida das formações de restinga do litoral norte da Bahia, Brasil. Acta Biológica Catarinense 1: 22-43.

IBGE (Instituto Brasileiro de Geografia e Estatística). 2004. Flora das Restingas do Litoral Norte da Bahia: Costa dos Coqueiros e Salvador. Projeto Flora/ Fauna - UE/BA - Microbiological Diagnosis: text and color atlas. Editora Médica e Científica Ltda Herbário Radambrasil, Salvador. Disponível em ftp://geoftp.ibge. gov.br/documentos/recursos_naturais/levantamento/ florarestinga.pdf/ (acesso em 01-XII-2014).

Kasecker, T.P., Silva, J.M.C., Rapini, A., RamosNeto, M.B., Andrade, M.J.G., Giulietti, A.M. \& Queiroz, L.P. 2009. Áreas-chave para espécies raras de fanerógamas. In: Giulietti, A.M., Rapini, A., Andrade, M.J.G., Queiroz, L.P. \& Silva, J.M.C. (eds.). Plantas raras do Brasil. Conservação Internacional, Belo Horizonte, pp. 433-471.

Lista de Espécies da Flora do Brasil. 2015. Jardim Botânico do Rio de Janeiro. Disponível em http:// floradobrasil.jbrj.gov.br/ (acesso em 15-IV-2015).

Martinelli, G. \& Moraes, M.A. 2013. Livro Vermelho da Flora do Brasil. Instituto de Pesquisas Jardim Botânico do Rio de Janeiro, Rio de Janeiro.

Mendes, K., Gomes, P. \& Alves, M. 2010. Floristic inventory of a zone of ecological tension in the Atlantic Forest of Northeastern Brazil. Rodriguésia 61: 669-676.

MMA (Ministério do Meio Ambiente). 2014. Mapeamento da cobertura vegetal dos biomas brasileiros. Disponível em http://www.mma.gov.br/estruturas/sbf chm rbbio/ arquivos/mapas_cobertura_vegetal.pdf/ (acesso em 01-XII-2014).
Oliveira-Filho, A.T. \& Carvalho, D.A. 1993. Florística e fisionomia da vegetação no extremo norte do litoral da Paraíba. Revista Brasileira de Botânica 16: 115-130.

Pereira, M.S.P. \& Alves, R.R.N. 2007. Composição Florística de um remanescente de Mata Atlântica na Área de Proteção Ambiental Barra do Rio Mamanguape, Paraíba, Brasil. Revista de Biologia e Ciências da Terra 7: 1-10.

Pessoa, E., Barros, F. \& Alves, M. 2014. Specklinia integripetala and S. ianthina spp. nov. (Orchidaceae Pleurothallidinae) from northeastern Brazil. Nordic Journal of Botany 32: 129-132.

Queiroz, E.P., Cardoso, D.B.O.L. \& Ferreira, M.H.S. 2012. Composição florística da vegetação de restinga da APA Rio Capivara, Litoral Norte da Bahia, Brasil Sitientibus série Ciências Biológicas 12: 119-141.

Radford, A.E., Dickison, W.C., Massey J.R. \& Bell, C.R. 1974. Vascular Plant Systematics. Harper \& Row Pub. New York.

Rodal, M.J.N., Lucena, M.F.A., Andrade, K.V.S.A. \& Melo, A.L. 2005. Mata do Toró: uma Floresta Estacional Semidecidual de terras baixas no nordeste do Brasil. Hoehnea 32: 283-294.

Silva, V.I.S. \& Menezes, C.M. 2012. Contribuição para o conhecimento da vegetação de restinga de Massarandupió, município de Entre Rios, BA, Brasil. Revista da Gestão Costeira Integrada 12: 239-251.

Stapf, M.N.S., Ranga, N.T. \& Silva, T.R.S. 2010. A new species of Cordia (Cordiaceae, Boraginales) from Brazil. Novon 20: 212-214.

Stehmann, J.R., Forzza, R.C., Salino, A., Sobral, M., Costa, D.P. \& Kamino, L.H.Y. 2009. Plantas da Floresta Atlântica. Jardim Botânico do Rio de Janeiro, Rio de Janeiro.

Tabarelli, M., Melo, M.D.V.C. \& Lira, O.C. 2006. A Mata Atlântica do nordeste. In: Campanili, M. \& Prochnow, M. (eds.). Mata Atlântica - uma rede pela floresta. RMA, Brasília, pp. 1-17.

Thiers, B. 2015. Index Herbariorum: A global directory of public herbaria and associated staff. New York Botanical Garden's Virtual Herbarium. Disponível em http://sweetgum.nybg.org/ih/ (acesso em 15-IV-2015).

Thomas, W.W., Jardim, J.G., Fiaschi, P., Neto, E.M. \& Amorim, A.M. 2009. Composição florística e estrutura do componente arbóreo de uma área transicional de Floresta Atlântica no sul da Bahia, Brasil. Revista Brasileira de Botânica 32: 65-78.

Udulutsch, R.G., Assis, M.A. \& Picchi, D.G. 2004. Florística de trepadeiras numa floresta estacional semidecídua, Rio Claro - Araras, Estado de São Paulo, Brasil. Revista Brasileira de Botânica 27: 125-134. 